\title{
Antiferromagnetism and spin glass in a Kondo lattice
}

\author{
S.G. Magalhães ${ }^{\mathrm{a}, 1}$, A.A. Schmidt ${ }^{\mathrm{b}, *}$, F.M. Zimmer ${ }^{\mathrm{a}}$, Alba Theumann ${ }^{\mathrm{c}}$, B. Coqblin ${ }^{\mathrm{d}}$ \\ - Departamento de Fisica-UFSM, Santa Maria, RS, 97105-900, Brazil \\ 'Depanamento de Matemática-CCNE, Lininersidade Federal de Santa Maria Faixa de Camobi, Km 9. \\ Santa Maria, RS, $97105 \cdot 900$, Brazil \\ Thatitu to de Ficica-UFRGS, Porto Aleore, RS 91501-970, Brozil \\ ${ }^{\mathrm{d}}$ Laboratoire de Physique des Solides. Universié Paris-Sud, Bàtiment 510. Orsay, 91405. France
}

\begin{abstract}
We study here the competition among the spin glass (SG), antiferromagnetism (AF) and the Kondo effect in a model which consists of two Kondo sublattices with both a Kondo coupling $J_{K}$ and a random interaction that couples the localized spins of different sublattices with antiferromagnetic mean $J_{0}$ and standard deviation $J$. The obtained phase diagram shows the sequence of phases $\mathrm{SG}, \mathrm{AF}$ and a Kondo state for increasing Kondo coupling $J_{\mathrm{K}}$ in qualitative agreement with the experimental data of $\mathrm{Ce}_{2} \mathrm{Au}_{1-x} \mathrm{Co}_{x} \mathrm{Si}_{3}$ alloys.

Keywords: Ising problem; Spin glass; Kondo lattice
\end{abstract}

The existence of disorder and frustration can be a source of non-trivial effects in Kondo lattice systems. For instance, several cerium and uranium disordered alloys have been found displaying a spin glass (SG) phase, antiferromagnetism (AF), ferromagnetism, non-Fermi liquid behavior and a Kondo state (see Ref. [1] and references therein). The sequence of phase transitions is quite dependent on the temperature range and doping in those alloys. Particularly, $\mathrm{Ce}_{2} \mathrm{Au}_{1-x} \mathrm{Co}_{x} \mathrm{Si}_{3}$ exhibits, at low temperature, a sequence $\mathrm{SG}-\mathrm{AF}$ and non-magnetic Kondo phase with increasing Cobalt concentration [2].

The aim of this paper is to present a mean field theory to describe the SG-AF phase transition in a model which consists of two Kondo sublattices with an intrasite Kondo-like exchange interaction and an intersite exchange inter action only between localized spins in different sublattices [3]. Moreover, there is no hopping of the conduction electrons between the two different

"Corresponding author. Tel.: +55-55-220-8854; fax: +5555-220-8032.

E-mail addresses: ggarcia@cenc.ufsm.br (S.G. Magalhães), alexia lana.ocne.ufsm.br (A.A. Schmidt).

'Also for correspondence. sublattices. The Hamiltonian of the model is

$$
\begin{aligned}
& H-\mu N=\sum_{p=\mathrm{A}, \mathrm{B}}\left[\sum_{i, j} \sum_{\sigma=\uparrow \downarrow} t_{i j} \hat{d}_{i, q, \sigma} \hat{d}_{j, p,}\right. \\
& \left.+\sum_{i} \delta_{0, p} \hat{n}_{i, p}^{f}+J_{K} \sum_{i}\left(\hat{S}_{i, p}^{+} \hat{s}_{i, p}^{-}+\hat{S}_{i, p}^{-} \hat{s}_{i p}^{+}\right)\right] \\
& +\sum_{i j} J_{i j} \hat{S}_{i, \mathcal{S}}^{c} \hat{S}_{j, \mathrm{~B}}^{\varepsilon}
\end{aligned}
$$

where $i$ and $j$ sums run over the $N$ sites of each sublattice. The intersite interaction $J_{i j}$ is a random variable with Gaussian distribution of mean $-2 J_{0} / N$ and variance $32 J^{2} / N$.

The spin variables are given in fermionic version as combinations of creation and destruction operators as defined in Refs. [4,5], but modified for the case of two Kondo sublattices. Therefore, the partition function can be given by using Grassmann variables for the localized fermions $\psi_{i, p, \sigma}$ and the conduction ones $\phi_{i, \sigma, \sigma}$ (where $p=\mathrm{A}, \mathrm{B})$. In the present approach, the static approximation is intensively used. The free energy can be obtained by using the replica trick. The method applied in the present approach, follows quite close to Refs. $[4,5]$ with the coupling between localized magnetic moments 


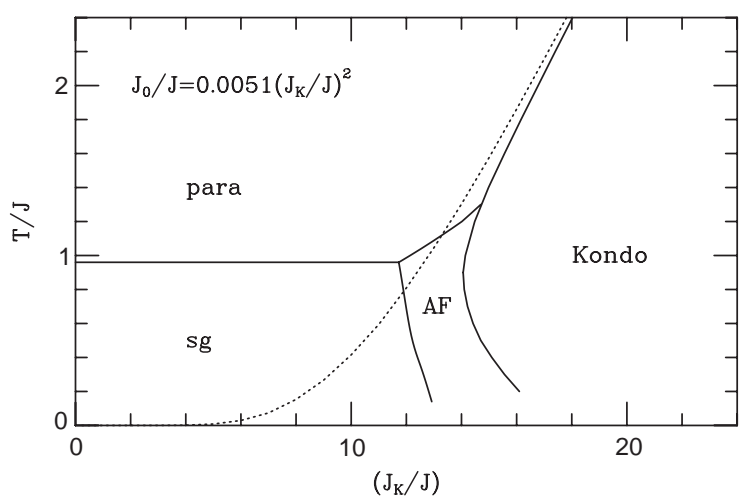

Fig. 1. The phase diagram $T / J$ versus $J_{\mathrm{k}} / J$ showing the sequences of phases SG (Spin Glass), AF (Antiferromagnetism) and a Kondo state. The variance $J$ is kept constant. The dotted line means the "pure" Kondo temperature.

treated like in Ref. [3] but in a fermionic version. Further details will be given elsewhere [6]. Therefore, the free energy can be found as

$$
\begin{aligned}
\beta F= & \beta J_{\mathrm{K}}\left(\left|\lambda_{\mathrm{A}}\right|^{2}+\left|\lambda_{\mathrm{B}}\right|^{2}\right)+\frac{\beta^{2} J^{2}}{2} \bar{\chi}_{\mathrm{A}} \bar{\chi}_{\mathrm{B}} \\
& +\frac{\beta^{2} J^{2}}{2}\left(\bar{\chi}_{\mathrm{A}} q_{\mathrm{B}}+\bar{\chi}_{\mathrm{B}} q_{\mathrm{A}}\right)-\frac{\beta J_{0}}{2} m_{\mathrm{A}} m_{\mathrm{B}} \\
& -\frac{1}{2} \int_{-\infty}^{\infty} \mathrm{D} \xi_{i_{\mathrm{A}}} \int_{-\infty}^{\infty} \mathrm{D} \xi_{j_{\mathrm{B}}} \\
& \times \ln \left[\prod_{p=\mathrm{A}, \mathrm{B}} \int_{-\infty}^{\infty} \mathrm{D} z_{p} \mathrm{e}^{E\left(h_{p}\right)}\right]
\end{aligned}
$$

with

$$
\begin{aligned}
E\left(h_{p}\right)= & \frac{1}{\beta D} \int_{-\beta D}^{\beta D} d x \ln \left[\cosh \left(\frac{x+h_{p}}{2}\right)\right. \\
& \left.+\cosh \sqrt{\left(\frac{x-h_{p}}{2}\right)^{2}+\beta^{2} J_{\mathrm{k}}^{2}\left|\lambda_{p}\right|^{2}}\right]
\end{aligned}
$$

where $h_{i, p}=\beta J\left[\sqrt{2 q_{p^{\prime}}} \xi_{i, p}+\sqrt{2 \bar{\chi}_{p^{\prime}}} z_{i, p}\right]-\beta J_{0} m_{p^{\prime}}\left(p \neq p^{\prime}\right)$. In our mean field description, the distinct regimes can be identified by the behaviour of the Kondo order parameter $\left|\lambda_{p}\right|$ and the internal field $h_{i, p}$ which depends on the spin glass order parameter $q_{p^{\prime}}$, the local susceptibility $\chi_{p^{\prime}}=\beta \bar{\chi}_{p^{\prime}}$ and the magnetization $m_{p^{\prime}}$. The solutions for the order parameters equations identify the phases: AF corresponding to $m_{\mathrm{A}}=$ $-m_{\mathrm{B}} \neq 0, \mathrm{SG}$ for $q_{p} \neq 0$ and a Kondo state given by $\left|\lambda_{p}\right| \neq 0$.

As the intrasite and the intersite exchange (see Eq. (1)) cannot be considered completely independent from each other [7], we introduce a relationship between $J_{0}$ and $J_{\mathrm{K}}$ : $J_{0} / J=\alpha\left(J_{\mathrm{K}} / J\right)^{2}$.

The results are given in a phase diagram $T / J$ versus $J_{\mathrm{K}} / J$ (see Fig. 1) which shows that increasing $J_{\mathrm{K}}$ favors the sequence of phase transitions $\mathrm{SG}-\mathrm{AF}-\mathrm{K}$ ondo state. If $J_{\mathrm{K}}$ is related to the cobalt doping in the alloy $\mathrm{Ce}_{2} \mathrm{Au}_{1-x} \mathrm{Co}_{x} \mathrm{Si}_{3}$, the phase diagram shown in Fig. 1 is in good agreement with the experimental sequence of phase transitions at low temperature [2]. Thus, our results can explain the sequence of phase transitions at low temperature for $\mathrm{Ce}_{2} \mathrm{Au}_{1-x} \mathrm{Co}_{x} \mathrm{Si}_{3}$ if we assume that it is valid to associate $J_{\mathrm{K}}$ with the cobalt doping and the relationship between $J_{\mathrm{K}}$ and $J_{0}$ given above.

\section{References}

[1] B. Coqblin, et al., Acta Phys. Pol. B 34 (2003) 1273.

[2] S. Majundar, et al., Solid State Commun. 121 (2002) 665.

[3] I.Ya. Korenblit, E.F. Shender, Sov. Phys. JETP 62 (1985) 1030.

[4] Alba Theumann, et al., Phys. Rev. B 63 (2001) 54409.

[5] S.G. Magalhães, et al., Eur. Phys. J. B 30 (2002) 419.

[6] S.G. Magalhães, et al., Eur. Phys. J. B 34 (2003) 447.

[7] J.R. Iglesias, et al., Phys. Rev. B 56 (1997) 11820. 